REVISTA PROYECCIONES N $N^{\circ} 12: 119-122$

Diciembre 1986 - ISSN 0716-0917

Jornada Matemáticas, Agosto 1986.

\title{
APLICACIONES DE LA TEORIA DEL MUESTREO
}

Carlos Vicuña Muñoz * y Alum.

\section{OBJETIVOS}

a) Utilización en casos prácticos de la Teoría del Muestreo.

b) Aplicabilidad de un Modelo de Respuesta Aleatorizada de variable de tipo continua con parámetros desconoci dos a la población de Antofagasta (Ingreso promedio de los hogares de estrato Socio-Económico alto).

DESCRIPCION

El presente trabajo se comprende de dos partes.

* Académico del Departamento de Aciministración y Economía. Universidad del Norte. Antofagasta. 
Las cuales son distintas en sus objetivos, intensidad y rigurosidad. Situación que se justifica por los objetivos centrales y particulares que guian la elaboración de cada una de las partes.

\section{Parte a)}

Dentro del programa de estudios de la carrera de Ingeniería Comercial de la Facultad de Economía de la Uni versidad del Norte, a nivel de tercer año se encuentra el Ramo de Estadística Empresarial, el cual tiene por objetivo: "Aplicación de técnicas estadísticas en: Administración de Negocios; Económica; Problemas Financieros, contables y otros.

En la primera parte del curso se estudia aplicaciones de técnicas muestrales. Y para la aprobación de esta parte se hace obligatorio la realización de un trabajo en donde se ocupen justamente la metodología de muestreo. En esta oportunidad se han seleccionado tres trabajos para ser presentados en este encuentro.

1) Estudio de la población de Edificios de Antofagasta.

Este trabajo trata de determinar la opinión que tienen los moradores de edificios de departamento con respecto a algunas situaciones como:

- Molestias más comunes.

- Privacidad.

- Desarrollo de la juventud.

- Entretenciones.

- otras. 
2) Grado de Aceptabilidad de la Universidad del Norte en los alumnos de cuarto medio de Antofagasta.

En este estudio se presentan los resultados estratificados de acuerdo a la situación del colegio. Si es fis cal, particular o subvencionado.

3) Audición de las radios en la ciudad de Antofagasta.

Este estudio es un intento de determinación de nivel de sintonía de las radio emisoras locales.

\section{Parte b)}

La constante evolución en las técnicas muestrales ha traído como consecuencia, un aumento en la disponibilidad de herramientas ofrecidas por la Teoría del Muestreo.

Una consecuencia de lo anterior es la creación de los Modelos de Respuesta Aleatorizada. Modelos que buscan solucionar los problemas que presenta la obtención de información de características internas o reservadas.

El respaldo técnico de estos modelos en consecuencia es fuerte y abundante, no así sus aplicaciones (básicamente por su reciente creación). La situación anterior moti va a un grupo de alumnos egresados de la carrera de Ingeniería Comercial a presentar como Memoria de Título el tema: "Obtención del ingreso promedio por hogar del estrato socioEconómico Alto de la Ciudad de Antofagasta por medio de un Mo delo de Respuesta Aleatorizada". 
La investigación arroja como resultados, aspectos y consideraciones que servirán de mucho en futuras aplica ciones de estos modelos.

Alumnos: Investigadores:- Alumnos Memoristas: Angélica Barrera Rojas

Thelma Palavicino Canales.

Alumnos de Ramo Estadística Empresarial. Juan Carlos Alvarez Chávez Guillermo Gaytan Cisternas. Juan Carlos Araya Pedraza

Miguel Angel Molina Zamudio

Carolina Tabilo Torrejón

Rolando Díaz Mundaca.

Hernán Mena Ramírez

Jaime oyanadel Meneses

Iván Soublette Mandiola. 